\title{
Identifying Key Developers using Artifact Traceability Graphs
}

\author{
H. Alperen Çetin \\ Bilkent University \\ Ankara, Turkey \\ alperen.cetin@bilkent.edu.tr
}

\author{
Eray Tüzün \\ Bilkent University \\ Ankara, Turkey \\ eraytuzun@cs.bilkent.edu.tr
}

\begin{abstract}
Developers are the most important resource to build and maintain software projects. Due to various reasons, some developers take more responsibility, and this type of developers are more valuable and indispensable for the project. Without them, the success of the project would be at risk. We use the term key developers for these essential and valuable developers, and identifying them is a crucial task for managerial decisions such as risk assessment for potential developer resignations. We study key developers under three categories: jacks, mavens and connectors. A typical jack (of all trades) has a broad knowledge of the project, they are familiar with different parts of the source code, whereas mavens represent the developers who are the sole experts in specific parts of the projects. Connectors are the developers who involve different groups of developers or teams. They are like bridges between teams.

To identify key developers in a software project, we propose to use traceable links among software artifacts such as the links between change sets and files. First, we build an artifact traceability graph, then we define various metrics to find key developers. We conduct experiments on three open source projects: Hadoop, Hive and Pig. To validate our approach, we use developer comments in issue tracking systems and demonstrate that the identified key developers by our approach match the top commenters up to $92 \%$.
\end{abstract}

\section{CCS CONCEPTS}

\section{- Software and its engineering $\rightarrow$ Programming teams.}

\section{KEYWORDS}

key developers, most valuable developers, developer categories, social networks, artifact traceability graphs, jack, maven, connector

\section{ACM Reference Format:}

H. Alperen Çetin and Eray Tüzün. 2020. Identifying Key Developers using Artifact Traceability Graphs. In Proceedings of the 16th ACM International Conference on Predictive Models and Data Analytics in Software Engineering (PROMISE '20), November 8-9, 2020, Virtual, USA. ACM, New York, NY, USA, 10 pages. https://doi.org/10.1145/3416508.3417116

\footnotetext{
Permission to make digital or hard copies of all or part of this work for personal or classroom use is granted without fee provided that copies are not made or distributed for profit or commercial advantage and that copies bear this notice and the full citation on the first page. Copyrights for components of this work owned by others than ACM must be honored. Abstracting with credit is permitted. To copy otherwise, or republish, to post on servers or to redistribute to lists, requires prior specific permission and/or a fee. Request permissions from permissions@acm.org.

PROMISE '20, November 8-9, 2020, Virtual, USA

(C) 2020 Association for Computing Machinery.

ACM ISBN 978-1-4503-8127-7/20/11 ..\$15.00

https://doi.org/10.1145/3416508.3417116
}

\section{INTRODUCTION}

Software development mainly depends on human effort. In a project, some developers take more responsibility, and the success rate of the project heavily depends on these developers. Thus, they are valuable and essential to develop and maintain the project, in other words, they are the key developers of the project.

Developers leave and join projects due to numerous reasons such as transferring to another project in the same company or leaving a company to work in another one. When a developer position is opened, it is filled by another developer in time. This is also known as developer turnover, which is a common phenomenon in software development and a critical risk for software projects [19]. It is more critical when the key developers leave the project. Therefore, identifying the valuable and indispensable developers is a vital and challenging task for project management. Developers can be valuable for a project in many different ways. All developers contribute to the project in some way or another. For instance, a developer may know a specific module very well, while another one knows a little related to multiple modules. In our study, similar to our previous work [7], we examine key developers under three categories: jacks, mavens and connectors.

Our motivation for this categorization comes from The Tipping Point by Gladwell [15]. The book discusses the reasons behind word-of-mouth epidemics. In The Law of Few chapter, the author justifies that three kinds of people are responsible for tipping ideas: connector, salesman and maven. Connectors have connections to different social groups, and they allow ideas to spread between these groups. Salesmen have a charisma that allows them to persuade people and change their decisions. Mavens have a great knowledge of specific topics and thus help people to make informed decisions.

Since there are traceable links among software artifacts as the connections among people in real life, we propose to use a similar categorization, connector and maven, as described in the book to find the key developers in a software project. A typical connector represents a developer who is involved in different (sub)projects or different groups of developers. Connecting divergent groups or (sub)projects increases this type of developers' significance because they connect the developers who are not in the same group (i.e. team) and touching different parts of a project means collective knowledge from different aspects of the project. Maven category represents the developers who are masters in details of specific modules or files in the project. Being the rare experts of specific parts of the source code makes these developers difficult to replace.

facks (of all trades) are the developers who have a broad knowledge of the project. They use or modify files from different parts of the project. Here, jack and connector definitions may interfere with each other since both define key developers who touch different parts of the project. To make it more clear, jack category purely focuses on knowledge when connector category focuses on 
connecting developers. "Jack" name comes from a figure of speech, jack of all trades, to define people "who can do passable work at various tasks" ${ }^{1}$. For the developers who have broad knowledge of projects, we use jack to remind this phrase.

To discover these three types of key developers, in this study, we address the following research questions (RQs):

RQ 1: How can we identify key developers in a software project?

RQ 1.1: How can we identify jacks in a software project?

RQ 1.2: How can we identify mavens in a software project?

RQ 1.3: How can we identify connectors in a software project?

In the following section, we share the related work. In Section 3 , we explain our methodology addressing the research questions. In Section 4, we share the details of the datasets and the important points of the preprocessing. In Section 5, we perform case studies in three different open source software (OSS) projects. In Section 6, we discuss the threats to validity of our study. In Section 7, we present our conclusions and possible future works.

\section{RELATED WORK}

In the literature, there are many studies on truck factor, developer roles and social developer networks. In the following, we mention them under separate sections.

\subsection{Truck Factor}

Truck factor (a.k.a. bus factor) is the answer to the following question: What is the minimum number of developers who have to leave the project before the project becomes incapacitated and has serious problems? To address this problem, Avelino et al. [4] associated files to authors by using the degree of authorship [14], then they found the minimum number of developers whose total file coverage is more than $50 \%$ of all files. Cosentino et al. [9] measured developers' knowledge on artifacts (e.g. files, directories and project itself) with different metrics such as "last change takes it all" and "multiple changes equally considered". They defined primary and secondary developers for the artifacts and proposed that the project will have problems with the artifact if all primary and secondary developers leave the project. Rigby et al. [23] studied a model on file abandonment. In their study, the author of a line is assigned by using git blame, and a file is abandoned when the authors of $90 \%$ of its lines left the project. They proposed to remove developers randomly until a specific amount of file loss occurs, and use the number of removed developers as the truck factor at that point.

Moreover, some researchers published empirical studies on existing truck factor algorithms. Avelino et al. [3] investigated abandoned OSS projects. In their definition, a project is abandoned when all truck factor developers leave. Ferreira et al. [12] performed a comparative study on truck factor algorithms and made a comprehensive discussion on them from many different viewpoints such as the accuracy of the reported results in the studies and the reasons why the truck factor algorithms fail at some circumstances.

\subsection{Developer Roles and Social Networks}

There has been a number of studies examined developer types from different perspectives. Kosti et al. [18] investigated archetypal

\footnotetext{
${ }^{1}$ https://www.merriam-webster.com/dictionary/jack-of-all-trades
}

personalities of software engineers. They chose extraversion and conscientiousness as their main criteria and focused on the binary combinations of them. Cheng and Guo [8] made an activity-based analysis of OSS contributors, then adopted a data-driven approach to find out the dynamics and roles of the contributors. Bella, Sillitti and Succi [11] classified OSS contributors as core, active, occasional and rare developers. Also, there are studies examined the core and periphery [10,16], hero [1], and key developers [20] in OSS projects. Likewise, Zhou and Mockus [28] claimed that Long Term Contributors (LTCs) are valuable for projects. They all have similar definitions, and in Section 5.3, we further discuss these studies by comparing with our study.

Besides developer types, researchers worked on communication networks of developers. Wu and Goh [27] studied the long term effects of communication patterns on success and performed experiments on how graph centrality, graph density and leadership centrality affect the success of OSS projects. Also, Kakimoto et al. [17] worked on knowledge collaboration through communication tools. They applied social network analysis to 4 OSS communities, and partially verified their hypothesis, which claims "Communications are actively encouraged before/after OSS released, especially among community members with a variety of roles but not among particular members"[17]. Moreover, Allaho and Lee [2] conducted a social networks (SN) analysis on OSS projects and found that OSS SNs follow a power-law distribution which means a small number of developers dominate the projects.

\section{METHODOLOGY}

To identify the key developers in a project, first we need to construct an artifact traceability graph as described in Section 3.1. Afterwards, from Section 3.2 to Section 3.4, we will explain our methodology for each corresponding research question.

\subsection{Artifact Traceability Graph}

Artifact traceability graphs include software artifacts and the connections between them. We denote nodes for software artifacts, which are developers, change sets (e.g. commits in Git), source files and issues. Then, we denote undirected edges for the relations (e.g. commit, review, include and linked) between those artifacts. For example, we add an edge for a commit relation between the developer node and the change set node if the developer is the author of the change set. The edges are undirected because reaching from one change set to another should be possible over the edges if they include the same file. Developers commit or review change sets. Change sets include a set of source files. Issues can be linked to a set of change sets and vice versa.

In the graph, we denote distances for each edge. Distances of the edges between developers and change sets are always zero (0) because these connections are there in order to keep track of who made commits and reviews. Other than commit and review cases, edge distances are calculated by using the recency of the bound change set. Our distance metric is inversely proportional to the recency of the change set. Recency and distance metrics are calculated as follows:

$$
\text { Recency }=1-\frac{\# \text { of days passed }}{\# \text { of days included to the graph }}
$$




$$
\text { Distance }=\frac{1}{\text { Recency }}
$$

Figure 1 shows a sample traceability graph, where the graph includes 300 days of the project history, and the numbers in the parentheses are the days that the commits are made. For example, CS3 was committed at the $90^{\text {th }}$ day (i.e. 210 days ago). All the edges of CS3 have the same distance, which is calculated as follows.

$$
\text { Distance }=\frac{1}{\text { Recency }}=\frac{1}{1-\frac{210}{300}}=\frac{1}{0.30}=3.33
$$

\subsection{Jacks (RQ 1.1)}

To find Jacks in a software project, we analyze the general knowledge of the developers on the project. By looking at the history of the project from its version control data, we can say that the source files keep the knowledge, in other words, the know-how of the project. There are studies to find the authors of source files (e.g. degree of authorship [14]). Authorship is not only about being the first author of the file but also about changing the source files in time depending on the recency of the change. In our study, we define reachability similar to this definition. If a developer can reach a file, $\mathrm{s}$ /he knows that file. Also, multiple developers can reach the same file at the same time. In the following, we explain how we find reachable files and file coverage for each developer.

3.2.1 Finding Reachable Files: We define reachable files of a developer as the files that are reached by the developer through the connections in the artifact traceability graph. For example, in Figure 1, D2 node can reach every file node in the graph through change sets, issues and other developers if there is no distance limit (i.e. a limit for the sum of distances on the edges in the graph). Actually, every developer can reach every file if the graph is connected and there is no distance limit. In that case, every developer would know every file, and we could not distinguish which developers know which source files. Also, assuming that all edges having the same importance might be problematic since some edges represent recent commits and reviews while others represent older ones. Our recency and distance definitions are utilized here to distinguish these types of situations. For example, the distance between CS3 and F4 is 3.33 while the distance between CS4 and F5 is 1.67, and there are around three months between the commit times of CS3 and CS4. Therefore, to handle these situations, we define the following rules:

(1) We need to set a threshold for distance while reaching from one node to another. For example, D2 cannot reach F5 if the threshold is 5 because $3.33+1.67+1.67=6.67$ and 6.67 is beyond the threshold 5 .

(2) One developer cannot reach files through other developers because it would transfer reachable files of a developer to another developer if the distance threshold is large enough For example, in Figure 1, D2 cannot reach F1 through D1, even if the distance threshold 308.33 or more.

Distance threshold is a parameter, and it depends on the distance formula given in Equation (2). Due to its nature, distance goes to infinity when recency goes to zero. In the graph, the oldest relations are the relations from the first day, and their edges have the highest distance. In this case, their distance is calculated as follows:

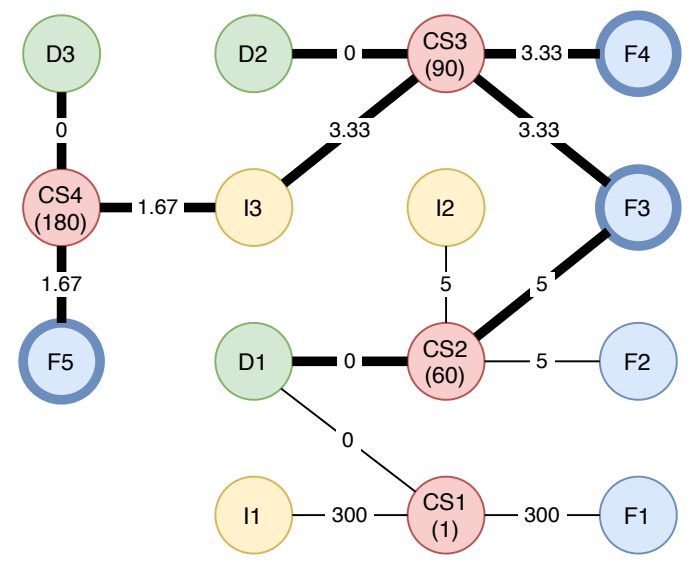

Figure 1: Sample artifact traceability graph. Some nodes and edges are highlighted to illustrate how the reachable files by D2 are found. (D: Developer, F: File, CS: Change Set, I: Issue)

$$
\text { Distance }=\frac{1}{\text { Recency }}=\frac{1}{1-\frac{299}{300}}=\frac{1}{\frac{1}{300}}=300
$$

Therefore, we need to set our threshold to 300 if we want to use every direct relation in the graph. Since almost all recently changed files are reachable by the developers who have recent commits in that case, using 300 as threshold would not enable us to distinguish which developers know which files.

We follow a simple way while deciding the distance threshold. In a 300-day graph, the edges with 0.1 or less recency belong to the change sets committed in the first 30 days. The rest of the graph corresponds to $90 \%$ of the time covered in the graph. Therefore, we can set the distance threshold to 10 , which allows us to use all direct relations from the last $90 \%$ of the days in the graph. Also, if we set it to $5,80 \%$ of the time would be covered. After this point, we continue with 10 as the distance threshold unless otherwise stated.

$$
\text { Distance }=\frac{1}{\text { Recency }}=\frac{1}{1-\frac{270}{300}}=\frac{1}{\frac{30}{300}}=\frac{1}{0.1}=10
$$

Figure 1 shows how reachable files for D2 are found in the sample graph. The highlighted files (F3, F4, F5) are reachable by D2. While finding these reachable files, we run a depth first search (DFS) algorithm starting from D2 with a stopping condition for reaching the distance threshold. The highlighted edges show the visited edges when DFS is started from D2 node. Also, the DFS algorithm does not go through another developer node. For example, the algorithm stopped when it encountered with the node of D1. Algorithm 1 shows the pseudo code for finding reachable files for each developer.

3.2.2 Identifying Jacks: While finding jacks, we sort the developers in descending order according to their file coverage in the software project. File coverage is simply the ratio of the number reachable files by the developer to the number of all files in the project, not just currently available files in the graph. Equation 3 shows the file coverage of some developer $d$.

$$
\text { File Coverage }_{d}=\frac{\# \text { of reachable files by } d}{\# \text { of all files in the project }}
$$



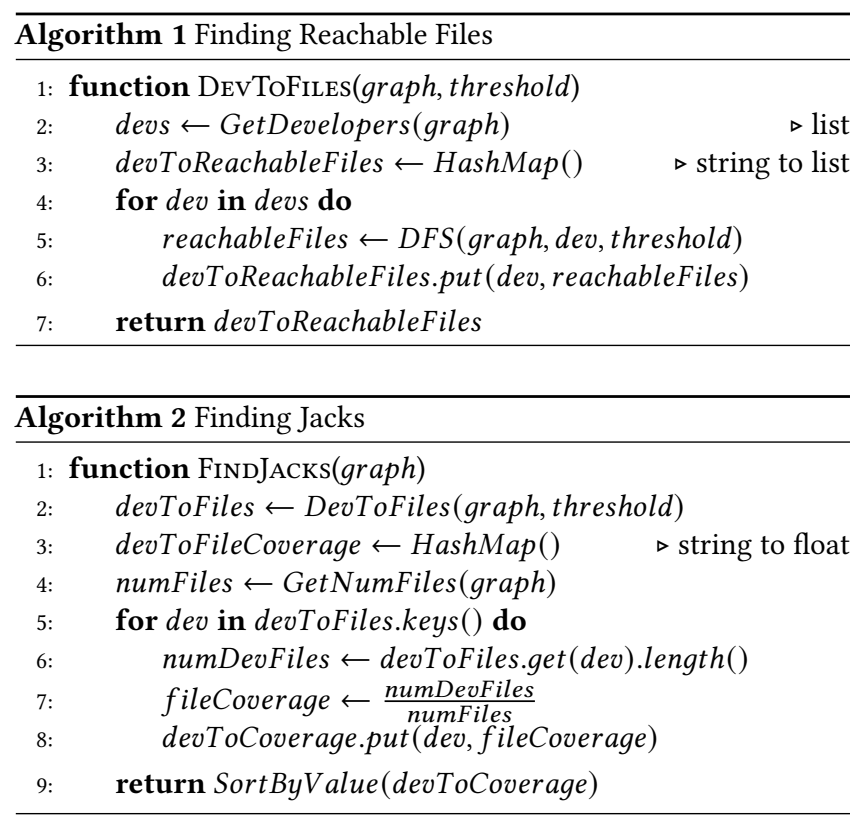

Table 1: Reachable files and file coverages for each developer in the sample artifact traceability graph

\begin{tabular}{ccc}
\hline Developer & Reachable Files & File Coverage \\
\hline D1 & F2 and F3 & $40 \%$ \\
D2 & F3, F4 and F5 & $60 \%$ \\
D3 & F3, F4 and F5 & $60 \%$ \\
\hline
\end{tabular}

Table 1 shows the reachable files and file coverages for each developer in the sample artifact traceability graph given in Figure 1. Algorithm 2 shows the pseudo code of finding jacks. First, it finds reachable files for each developer, then calculates file coverage scores for developers. Finally, it returns developers in descending order according to their file coverage scores.

\subsection{Mavens (RQ 1.2)}

By definition, mavens are the rare experts of specific parts, files or modules of the project. As we stated in Section 3.2, the source files in a software project are the reflection of the knowledge (i.e. know-how). Since mavens are the rare expert developers on specific parts, they have knowledge that the others do not have. Thus, we need to find lesser-known parts of the project.

3.3.1 Rarely Reached Files: First, reaching a file through the edges in the artifact graph means knowing the file. To meet the maven definition, we can use the files only reached by a limited number of developers. We call this type of files rarely reached files, and we set this limit to 1 , which means that the files reached by only one developer are the rarely reached files. This could be a configurable parameter according to the size of the project. For example, for the graph given in Figure 1, F2 is a rarely reached file. Actually it is the only one as it can be seen in Table 1 .

Algorithm 3 shows how to find rarely reached files. It is assumed that devToRareFiles is initialized with developer names and empty
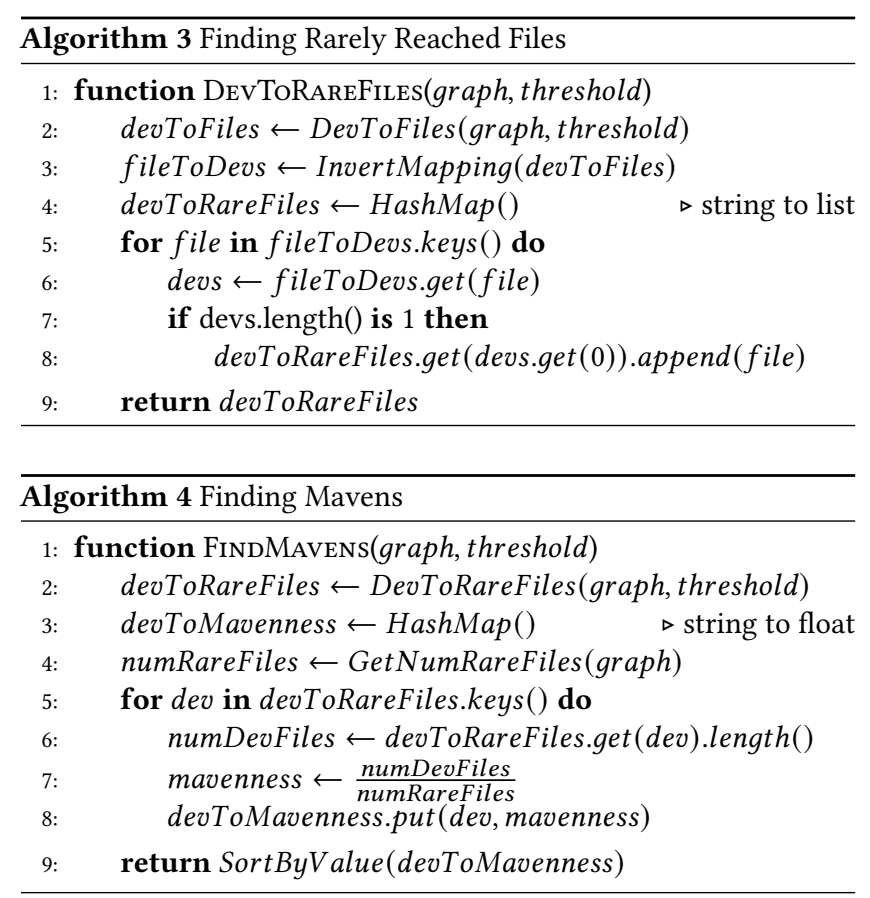

lists. Also, InvertMapping function generate a mapping from values to keys. For instance, it inverts the hashmap $\{D 1:[F 1], D 2$ : $[F 1, F 2]\}$ to the hashmap $\{F 1:[D 1, D 2], F 2:[D 2]\}$.

3.3.2 Identifying Mavens: To find mavens, we consider the number of the rarely reached files of the developers. For a better comparison among developers, we define mavenness of a developer $d$ as follows:

$$
\text { Mavenness }_{d}=\frac{\# \text { of rarely reached files of } d}{\# \text { of all rarely reached files }}
$$

While finding mavens, first we find reachable files as explained in Section 3.2.1, then we find rarely reached files as explained in Section 3.3.1 and given in Algorithm 3, finally we calculate mavenness scores and sort the developers according to them in descending order. Algorithm 4 shows the procedure.

\subsection{Connectors (RQ 1.3)}

Connectors are the developers who are involved in different subprojects or teams. The main idea behind the connector definition is connecting developers who have no other connections, in other words, being the bridge between different groups of developers. Using node centrality, we identify this type of developers on artifact traceability graphs defined in Section 3.1.

3.4.1 Calculating Betweenness Centrality: Betweenness centrality of a node is based on the number of shortest paths passing through that node. Freeman [13] discussed that betweenness centrality is related to control of communication. Also, Bird et al. [5] used betweenness centrality to find the gatekeepers in the social networks of mail correspondents. Therefore, we hypothesize that betweenness centrality can be a measure to find connectors. Betweenness centrality of some node $v$ where $V$ is the set of nodes, $s$ and $t$ are some nodes other than $v$ : 


$$
c_{B}(v)=\sum_{s \neq v \neq t \in V} \frac{\# \text { of shortest paths passing through } v}{\text { \# of shortest }(s, t) \text {-paths }}
$$

For a better comparison among developers, betweenness values are normalized with $2 /((n-1)(n-2))$ where $n$ is the number nodes in the graph. For betweenness centrality related operations, we use NetworkX package ${ }^{2}$, which uses faster betweenness centrality algorithm of Brandes [6].

To use betweenness centrality, we need a graph composed of only developers because we are looking for developers who connect other developers to each other. Sulun et al.[26] proposed a metric, know-about, to find how much developers know the files. They found different paths between the files and the developers in the artifact graph, and defined know-about as the summation of the reciprocals of the path lengths. Similarly, we propose to use different paths between developers to find how much they are connected in the artifact graph. The next section explains the details.

3.4.2 Constructing the Developer Graph: Developer graph is a projection of the artifact traceability graph. It defines distances directly between developers in a different way, not as we mentioned in Section 3.1. When projecting an artifact graph to a developer graph, we find all different paths between each developer pair with a depth limit of 4 . Since connector definition is not about knowing the files but about connecting the other developers, recency is not a concern and it is assumed that all edges in the artifact graph have the same distance of 1 . Thus, depth limit 4 means that the maximum path length can be 4 . Therefore, in the traceability graph, two developers can be connected through the paths with length of 2 (through a change set node), or the paths with length of 4 (through 2 different change set nodes and a file node connected to them). These kinds of paths can be seen in the sample graph in Figure 2. We find the paths between two developers through software artifacts, not through other developers. For example, in Figure 2, there is a path between D2 and D3 through D1, but we interpret this path as the combination of two paths: D1-D2 and D1-D3. Since the developers in the same team potentially work on the same group of files and these files will be close to each other (they will be connected through change sets because they will be changed by the same group frequently) in the traceability graph, the method mentioned above finds the paths between the developers in the same group. So, the developers who have connections in different groups will be favored in betweenness centrality calculations.

After finding the paths between each developer pair, we define a new distance metric, Reciprocal of Sum of Reciprocal Distances (RSRD). We define RSRD as follows when $D$ denotes the set of all distances between two developers (i.e. the set of lengths of all different paths between two developers) and $d$ is a distance in $D$ :

$$
R S R D=\left[\sum_{d \epsilon D} d^{-1}\right]^{-1}
$$

Reciprocals of distances make larger contributions to the score for closer nodes. For example, $\frac{1}{2}>\frac{1}{4}$ and the path with length of 2 make a larger contribution. After summing the contributions of

\footnotetext{
${ }^{2}$ https://networkx.github.io/
}

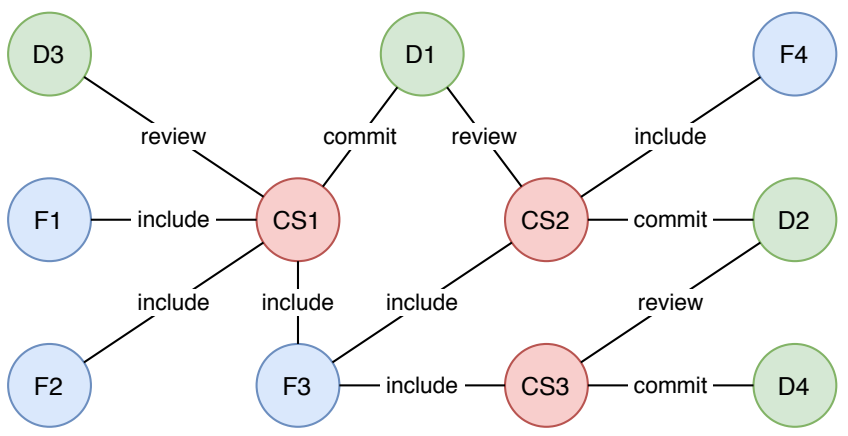

Figure 2: Another sample artifact traceability graph. (D: Developer, F: File, CS: Change Set)

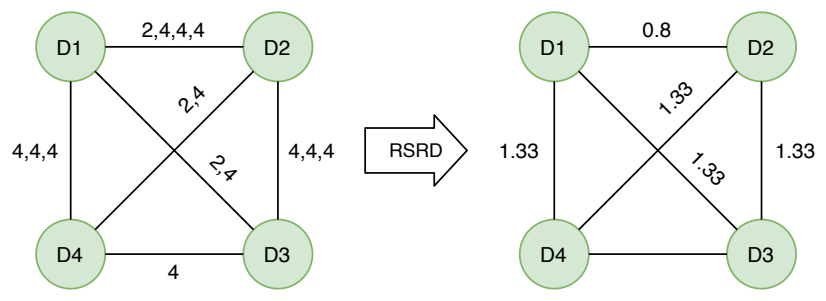

Figure 3: Sample developer graph. (D: Developer)

all reciprocal distances, larger values represent a stronger connection. For example, the first one means a stronger connection when the first one is $\frac{1}{2}+\frac{1}{4}=\frac{3}{4}$ and the second one is $\frac{1}{4}+\frac{1}{4}=\frac{2}{4}$. To use betweenness centrality, we need to inverse the result of this summation, because the nodes with stronger connections need to be closer. For example, for the numbers in the previous example, $\frac{4}{3}=1.33$ is smaller than $\frac{4}{2}=2$, and it means a closer relation. At the end, a smaller RSRD score represents a closer relationship between two developers, just like any other distance metric.

Figure 3 shows how the developer graph is constructed from the sample artifact graph in Figure 2. For example, $(2,4,4,4)$ are the distances of the different paths between D1 and D2 in Figure 2, and the RSRD between these two developers is calculated as follows:

$$
\left(2^{-1}+4^{-1}+4^{-1}+4^{-1}\right)^{-1}=\left[\frac{1}{2}+\frac{1}{4}+\frac{1}{4}+\frac{1}{4}\right]^{-1}=\left[\frac{5}{4}\right]^{-1}=0.8
$$

Algorithm 5 shows the pseudo code for calculating RSRD for a given graph and depth limit. First, it runs a DFS algorithm starting from each developer to find the paths to other developers. Second, it removes duplicates because the DFS results include paths for two ways. For example, DFS finds paths for both $(D 1, D 2)$ and $(D 2, D 1)$, and it removes $(D 2, D 1)$. Then, for each developer pair, it calculates their RSRD value by using the length of the paths.

3.4.3 Identifying Connectors: When identifying connectors, we use the betweenness centrality of developers in the developer graph. Algorithm 6 shows the procedure. First, it finds different paths and RSRD values for each developer pair as mentioned above. Then, it creates a developer graph with these RSRD values and finds betweenness centrality for each developer in that graph. Finally, it sorts developers in descending order according to their centrality. 

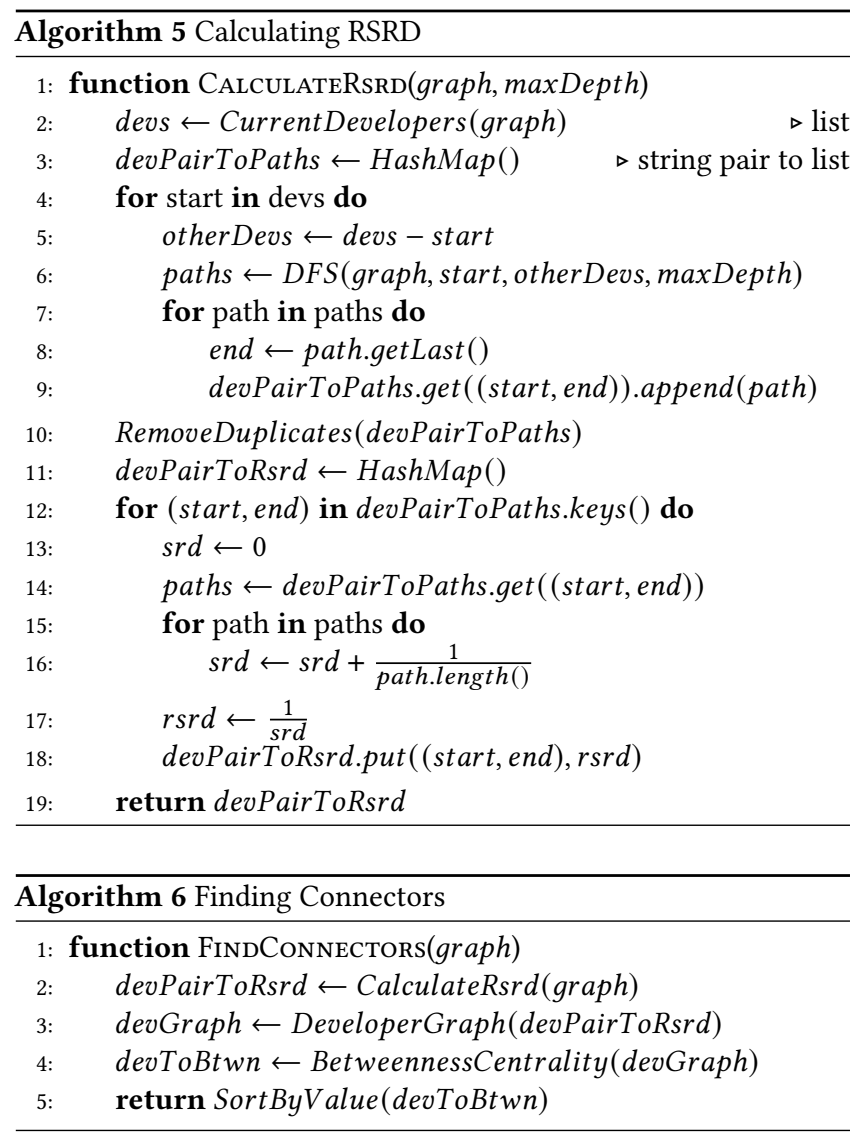

\section{DATASET}

\subsection{Selecting Datasets}

As we mentioned before, we use software artifacts from project history to construct the artifact traceability graph. More specifically, our approach needs change sets (i.e. commits) and their related data such as author, changed files and linked issues. Rath and Mader [21] published datasets for 33 OSS projects, SEOSS 33. All 33 datasets are available online ${ }^{3}$. Out of 33 projects, we selected Apache Hadoop ${ }^{4}$, Apache Hive ${ }^{5}$ and Apache Pig ${ }^{6}$ since these three projects have the highest issue and change set link ratios among SEOSS 33 datasets. The datasets include data from version control systems (e.g. Git) and issue tracking systems (e.g. Jira). Table 2 shows the details for each dataset with a varying number of issues and change sets.

\subsection{Preprocessing}

Data is already extracted from the version control and issue tracking platforms and provided in an SQL dataset. Nonetheless, we processed the data in order to prevent errors and calculate specific fields. We did not use all information in the dataset; change_set, code_change and change_set_link tables were enough to create nodes for developers, changes sets, issues and files.

\footnotetext{
${ }^{3}$ https://bit.ly/2wukCHc

${ }^{4}$ https://hadoop.apache.org/

${ }^{5}$ https://hive.apache.org/

${ }^{6}$ https://pig.apache.org/
}

We processed change sets from change_set table ordered by commit_date, extracted the data required and dumped them into a file as JSON formatted string of change sets in the temporal order. For each change set, we extracted the following information: commit hash, author, date (commit_date), issues linked, set of file paths with their change types, number of files in the project (after the change set).

In the data extraction, the following points are important:

- We only extracted the code changes in java files which, we assumed, end with ".java" extension. If a change set has no code change including a java file, we ignored it completely.

- We ignored merge change sets (is_merge is 1) since they could inflate the contributions of some developers [1].

- We created a look-up table for each project to detect different author names of the same authors. They are created manually by looking at the developer names and their email addresses. For example, "John Doe" and "Doe John" can be the same developer if they share a common email address. We used this table to correct the author names by replacing them.

- In order to calculate mavenness score (See Section 3.3), we needed the number of files in the project after each change set. Thus, we tracked the set of current files over time. After each code change, we removed the file if its change type is DELETE, and we added the file if its change type is $A D D$.

- Git does not track RENAME situations explicitly, and the dataset [21] did not share such information about the code changes. When a file is renamed, it is a DELETE and an $A D D$ for Git (if there is no change in the file) ${ }^{7}$. In the code_change table, there are three change_types: ADD, DELETE and MODIFY. In that case, we needed to handle renames because it would affect our traceability graph and change the knowledge balance among developers. We treated (DELETE, ADD) pairs in the same change set (commit) as RENAME when the following conditions were satisfied:

- Both have the same file name (file paths are different).

- The number of lines deleted in DELETE code change and the number of lines added in $A D D$ code change are equal. So, our rename algorithm only detects file path changes and does not check file contents. For example, it detects RENAME when "example.java" moved from "module1/example.java" to "module2/example.java" but does not detect it when the file content is changed. Since we used the datasets from SQL tables [21] directly and we did not mine them from Git repositories ourselves, we used the heuristic give. above.

- In Hadoop dataset, we detected that there were duplicate commits. Even though the commit hashes were different, the rest of the extracted data were identical. This situation only applies for Hadoop, the same preprocessing steps did not produce such a situation for Hive and Pig. We removed these change sets by using string comparison for all parts of the JSON string except the commit hash.

Table 2 show the number of change sets for each dataset after preprocessing. Also, we share our implementation online ${ }^{8}$, and it includes the preprocessing script.

\footnotetext{
${ }^{7}$ https://git-scm.com/book/en/v2/Git-Basics-Recording-Changes-to-the-Repository ${ }^{8}$ https://github.com/hacetin/KeyDev-PROMISE20/
} 
Table 2: Dataset Details

\begin{tabular}{c|cccc|ccc}
\hline \multirow{2}{*}{ Project } & \multicolumn{4}{|c|}{ Before Preprocessing [21] } & \multicolumn{3}{c}{ After Preprocessing } \\
\cline { 2 - 8 } & $\begin{array}{c}\text { Time Period } \\
\text { (months) }\end{array}$ & $\begin{array}{c}\text { \# of } \\
\text { Issues }\end{array}$ & $\begin{array}{c}\text { \# of } \\
\text { Change } \\
\text { Sets }\end{array}$ & $\begin{array}{c}\text { Linked Change } \\
\text { Sets to a Set } \\
\text { of Issues (\%) }\end{array}$ & $\begin{array}{c}\text { \# of } \\
\text { Change } \\
\text { Sets }\end{array}$ & $\begin{array}{c}\text { \# of Change Sets } \\
\text { added or modified } \\
\text { files more than 10 }\end{array}$ & $\begin{array}{c}\text { \# of Change Sets } \\
\text { added or modified more than 50 } \\
\text { files mon }\end{array}$ \\
\hline Hadoop & 150 & 39,086 & 27,776 & 97.13 & 15178 & $1900(12.5 \%)$ & $129(0.8 \%)$ \\
Hive & 113 & 18,025 & 11,179 & 96.34 & 9030 & $1062(11.8 \%)$ & $127(1.4 \%)$ \\
Pig & 123 & 5234 & 3134 & 92.85 & 2401 & $240(10.0 \%)$ & $32(1.3 \%)$ \\
\hline
\end{tabular}

\subsection{Handling Large Change Sets}

Change set means a set of file changes, and it is called large change set when the number of changed files is more than a specific number. For example, initial commits of a project most probably include many files, and it is a typical example for large commits. Another example is moving a project into another project. In that case, its change set includes all the files in the added project.

Committing a large number of files in one change set is not considered to be a good practice in software engineering. Sadowski et al.[25] claim that $90 \%$ of the changes in Google modify less than 10 files. Also, Rigby and Bird [22] excluded the changes that contain more than 10 files in their case studies. In our experiments, we use a looser limit for excluding change sets. In the following, we explain the details on removing the large change sets:

- Regardless of the size of a change set, we apply changes to the traceability graph for DELETE and RENAME types. Since the knowledge of deleted files is not required after that point and renamed files need to proceed with their new names.

- If a change set includes more than 50 files which are added or modified, we ignore these $A D D$ and $M O D I F Y$ code changes. We did not use 10 as the limit because we did not want to lose $10.0-12.5 \%$ of the datasets (See Table 2). Also, sometimes large commits can exist even though it is not a good practice. Our purpose is handling initial commits of the projects and project movements. So, choosing 50 is a good trade-off for the limit of the number of files added or modified in a change set. It is not that small to cause losing $10.0-12.5 \%$ of the datasets and not that large to include commits like initial commits.

\section{CASE STUDIES}

\subsection{Experimental Setup}

In the experiments, we used NetworkX package ${ }^{2}$ for graph operations. In order to prevent potential bugs, we used its built-in functions whenever possible (e.g. calculating betweenness centrality, finding paths between developers). However, we implemented the DFS algorithm for reachable files in Algorithm 1 because it was very specific to our case (e.g. the stopping condition is different). Our source code is available online ${ }^{8}$.

How much time the artifact graph should cover is a parameter in our method. We chose a sliding window approach over an incremental window in time, in other words, the artifact graph always includes the change sets committed in a constant time period. Followings are the reasons behind this choice:

- If the time period of the graph changes over time, the meaning of the recency changes. For example, 0.9 recency means

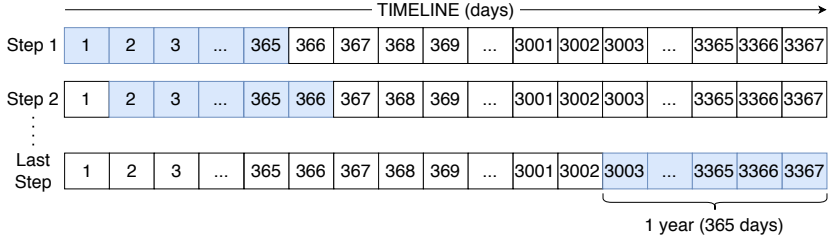

Figure 4: Experimental Setup

30 days ago in a 300-day graph while the same recency corresponds to 50 days ago in a 500-day graph. Thus, keeping the time period (sliding windows size) constant enables recency scores to have the same meaning in different time points.

- Keeping every artifact from history enlarges the graph every day, and the algorithms run slower in larger graphs. Therefore, removing unnecessary parts (the artifacts older than one year) means less run time.

- In OSS projects, there is no data about leaving developers. If we keep every artifact from the history, we should calculate scores even for former developers. Therefore, removing old artifacts enables us to keep track of the current developers. If the graph keeps the last 365 days, we assume the developers who contributed to the project in the last 365 days are the current active developers.

We used a 1-year (365 days) sliding window in our experiments. Figure 4 shows how the included days change in iterations. The numbers on the figure come from the Hive dataset. "3367 days" corresponds to the number of days after preprocessing. There are 3003 iterations including the initial window. We tracked the dates over change sets. When forwarding the window one day, firstly, we removed the change sets of the first day of the window. Then, we added the change sets of the day after the last day of the window. For each iteration, we calculated scores for jacks, mavens and connectors, then, we reported them and their scores in descending order. The same procedure was repeated for Hadoop and Pig.

\subsection{Results}

Since we propose to use jack, maven and connector as the key developer categories for the first time and there is no classification of developers for these types in the literature, we are not able to compare our approach with others. Also, since we conducted our experiments on OSS projects, we have no data for developer labels for these projects. However, we can show that the results of our approach are compatible with other statistics of the projects.

To validate our approach, we propose to use developers' comments on issues. facks are the developers who have broad knowledge by definition, and we identified them by finding their file 
Table 3: Mean accuracy percentages for the key developers of our approach vs. the developers selected randomly in Monte Carlo simulation

\begin{tabular}{|c|c|c|c|c|c|c|c|c|c|c|}
\hline \multirow{2}{*}{$\begin{array}{c}\text { Key Developer } \\
\text { Category }\end{array}$} & \multirow{2}{*}{ Projects } & \multirow{2}{*}{$\begin{array}{c}\text { Top } \\
\text { Commenters }\end{array}$} & \multicolumn{4}{|c|}{ Key Developers } & \multicolumn{4}{|c|}{ Randomly Selected Developers } \\
\hline & & & Top-1 & Top-3 & Top-5 & Top-10 & Top-1 & Top-3 & Top-5 & Top-10 \\
\hline \multirow{12}{*}{ JACKS } & \multirow{4}{*}{ HADOOP } & Top-1 & 6.82 & 19.04 & 27.10 & 50.47 & 1.97 & 5.88 & 9.79 & 19.56 \\
\hline & & Top-3 & - & 22.65 & 30.20 & 47.81 & - & 5.58 & 9.30 & 18.59 \\
\hline & & Top-5 & - & - & 29.62 & 47.40 & - & - & 9.60 & 19.18 \\
\hline & & Top-10 & - & - & - & 41.75 & - & - & - & 19.14 \\
\hline & \multirow{4}{*}{ HIVE } & Top-1 & 44.16 & 71.43 & 81.65 & 92.21 & 6.24 & 18.73 & 31.23 & 58.17 \\
\hline & & Top-3 & - & 54.78 & 70.20 & 84.12 & - & 17.46 & 29.10 & 54.17 \\
\hline & & Top-5 & - & - & 57.02 & 73.28 & - & - & 26.60 & 49.53 \\
\hline & & Top-10 & - & - & - & 55.31 & - & - & - & 38.84 \\
\hline & \multirow{4}{*}{ PIG } & Top-1 & 59.16 & 86.23 & 88.90 & 89.91 & 11.77 & 35.41 & 56.35 & 83.55 \\
\hline & & Top-3 & - & 75.27 & 85.26 & 89.99 & - & 34.95 & 55.61 & 83.05 \\
\hline & & Top-5 & - & - & 66.91 & 79.66 & - & - & 46.61 & 73.73 \\
\hline & & Top-10 & - & - & - & 59.15 & - & - & - & 55.84 \\
\hline \multirow{12}{*}{$\begin{array}{c}\text { INTERSECTION } \\
\text { OF ALL } \\
\text { (Sorted by } \\
\text { Jack Score) }\end{array}$} & \multirow{4}{*}{ HADOOP } & Top-1 & 7.22 & 21.33 & 34.97 & 59.04 & 1.96 & 5.87 & 9.77 & 17.48 \\
\hline & & Top-3 & - & 23.80 & 32.68 & 52.05 & - & 5.57 & 9.28 & 16.65 \\
\hline & & Top-5 & - & - & 31.26 & 49.08 & - & - & 9.59 & 17.16 \\
\hline & & Top-10 & - & - & - & 44.38 & - & - & - & 17.04 \\
\hline & \multirow{4}{*}{ HIVE } & Top-1 & 56.94 & 75.92 & 81.15 & 87.98 & 6.23 & 10.92 & 12.50 & 14.68 \\
\hline & & Top-3 & - & 49.40 & 55.17 & 61.33 & - & 10.38 & 11.90 & 14.01 \\
\hline & & Top-5 & - & - & 38.86 & 44.94 & - & - & 10.79 & 12.68 \\
\hline & & Top-10 & - & - & - & 30.48 & - & - & - & 10.70 \\
\hline & \multirow{4}{*}{ PIG } & Top-1 & 66.26 & 86.41 & 86.63 & 86.63 & 11.78 & 22.36 & 23.58 & 23.58 \\
\hline & & Top-3 & - & 55.54 & 56.30 & 56.30 & - & 22.44 & 23.78 & 23.78 \\
\hline & & Top-5 & - & - & 39.39 & 39.39 & - & - & 20.73 & 20.73 \\
\hline & & Top-10 & - & - & - & 21.29 & - & - & - & 15.45 \\
\hline
\end{tabular}

coverage in the project. Therefore, the jacks should be involved in the issues such as bugs and enhancements more than other developers. We claim that, by definition, the top jacks and the top commenters in the project's issue tracking system (e.g. Jira) should be mostly the same developers. However, we cannot claim that mavens and connectors should be among the top commenters. To validate the results of these categories, we offer to use the developers who are jack, maven and connector at the same time, in other words, the intersection of all kinds of key developers. In that way, we include mavens and connectors to our validation, and we show how the intersection developers overlap with the top commenters. While using the intersection developers, we sorted them by their jack score (i.e. file coverage) since we cannot combine betweenness centrality, mavenness score and file coverage properly. So, in the case studies, we examined the jacks and the intersection developers.

The datasets [21] we used for experiments include data from issue tracking systems (e.g. Jira). In the change_set_link table, there are links between issues and change sets, which means we can use the comments made to issues in the traceability graph. The datasets supply the display_name of the commenters in issue_comment table. The names in the display_name field matches the developer names in author field of change_set table. So, we can find which developers made how many comments to the issues in the graph. To increase the validity of the number of comments for each developer, we corrected the commenter names by using the look-up table created manually in preprocessing (See Section 4.2).
The Key Developers column in Table 3 shows the accuracy of our approach when we treat the top commenters as the actual key developers (i.e. ground truth). "Top commenters" means a ranked list of commenters according to the number of comments that they made to the issues in the last year. The predicted key developers by our model are consistent with the top commenters up to $92 \%$.

Accuracy is calculated as shown in Equation 7, where $K D$ is the ranked list of key developers, $C$ is the ranked list of commenters, $D$ is the set of dates (i.e. days or iterations in Figure 4) and k refers the numbers in Top-k phrases in the Table 3. For example, the accuracy of day $d$ for (Top-3, Top-5) cell is calculated as follows: If $C_{d}(3)=\{D 1, D 2, D 3\}$ and $K D_{d}(5)=\{D 1, D 2, D 4, D 5, D 6\}$, the accuracy is $\frac{|\{D 1, D 2\}|}{|\{D 1, D 2, D 3\}|}=\frac{2}{3}=0.67$.

$$
\text { Mean Accuracy }\left(k_{1}, k_{2}\right)=\frac{1}{|D|} \sum_{d}^{D} \frac{\left|C_{d}\left(k_{1}\right) \cap K D_{d}\left(k_{2}\right)\right|}{\left|C_{d}\left(k_{1}\right)\right|}
$$

Since there is no comparable approach that finds our subcategories of key developers, we used the Monte Carlo simulation as a baseline approach. We randomly selected the key developers for each day from the existing developers in the graph, in other words, from the developers who committed changes to the source code in the sliding window period. While producing random developers, we considered the number of key developers in our results since the simulation should provide random results in the same structure. For example, we selected 4 random developers if our approach 
found 4 jacks in that day even if $k_{2}$ is 5 . Then, we calculated mean accuracies in the same way shown in Equation 7. This experiment with random key developers is repeated 1000 times. The Randomly Selected Developers column in Table 3 shows the average of the accuracies of 1000 simulations. It can be seen that our approach is more successful than the random model for all cases.

Hadoop, Hive and Pig have different scales. Pig is a small project with tens of developers while the others have hundreds of developers in their whole history. Even though they both have hundreds of developers and their time periods are not that different, Hadoop has a lot more change sets and issues than Hive as shown in Table 2. The average number of active developers for each project, in other words, the average number of developers in the traceability graph over time are as follows: 9.05 in Pig, 35.08 in Hive and 49.86 in Hadoop. So, it is clear that Hadoop is a more active project than Hive. Also, the differences between the results of projects in Table 3 infer the same conclusion. Both the results of our algorithms and the results of Monte Carlo simulation show that the more active developers exist, the harder it becomes to predict key developers. Even though the accuracies are different among the projects due to the fact mentioned above, our results are better than the results of the random model for all cases. Also, the key developers predicted by our approach and the top commenters overlap up to $92 \%$.

\subsection{Discussion}

In this section, we discuss the studies which propose a definition for core, hero, key or LTC developers similar to our definition.

Agrawal et al. [1] worked on hero developers in software projects. According to their definition, a project has hero developers if $20 \%$ of the developers made $80 \%$ of the contributions. Hero developers are similar to key developers in our case, as projects mostly depends on them. The authors provided a definition for the projects with hero developers, then analysed hero and non-hero projects without providing a validation. In our study, we subcategorized the key developers into three categories and provided separate algorithms to identify key developers.

Oliva et al. [20] worked on characterizing the key developers, that is "the set of developers who evolve the technical core". First, they detected the commits to core files in the project. Then, they ranked the developers according to their core commit counts, and considered first $25 \%$ of the list as key developers. Without validating their key developers, they analyzed the identified key developers in terms of contribution characteristics, communication and coordination within the project. Also, they only performed an experiment on a small project with 16 developers. In our study, we investigated our algorithms in three projects different in size, and validated our key developers using top commenters in issue tracking systems.

Crowston et al. [10] examined the core and periphery of OSS team communications. They analyzed if the three following methods produce similar results or not: the contributors named officially (e.g. support manager, developer), the contributors who contribute the most to the bug reports, and the contributors who are defined by a pattern of interactions in bug tracking systems. Since they used data from SourceForge ${ }^{9}$, they had access to official labels of the contributors. In our case, Git repositories does not provide any

\footnotetext{
${ }^{9} \mathrm{https}: / /$ sourceforge.net/
}

labels for developer roles in the project and Apache organization only provide the full list of current developers.

Joblin et al.[16] studied core and peripheral developers. The core developers are the essential developers in the projects as the key developers in our study. They worked on count-based (e.g. number of commits as in [1]) and network-based (e.g. degree centrality in developer graph from version control systems and mailing lists) metrics. They established a ground truth by making a survey with 166 participants. We were not able to examine their data because the project and survey data links are not accessible through their website ${ }^{10}$. Also, their survey shows the actual core developers for a snapshot of the time, while our approach provide results continuously with a sliding window approach.

Zhou and Mockus [28] defined an LTC as "a participant who stays with the project for at least three years and is productive". They claimed that LTCs are crucial for success of the projects. They mainly investigated how a new joiner become an LTC (i.e. a valuable contributor). In our study, we investigated how to find key developers rather than examining how new joiners evolve in time.

\section{THREATS TO VALIDITY}

Construct validity is about how the operational measures in the study represent what is investigated according to the RQs [24]. We used a dataset from another study[21], and their mining process can potentially affect our results. To reduce the threat caused by the data mining process, we eliminated the possible problems (e.g. we corrected author names manually by looking at their names and email addresses.) in preprocessing (See Section 4.2). However, there might still be problems related to data integrity.

Internal validity concerns if the causal relations are examined or not [24]. While building the graphs and defining algorithms, we made many decisions related to thresholds including choosing 50 as the limit for the number of files added or modified in a change set, choosing 10 as the distance threshold in file reachability and using 365-day (1-year) sliding window in the experiments. We tried various options and made the final decisions after evaluating their results. In the corresponding sections of this study, we shared the justifications behind these decisions. For example, we chose 10 as the distance threshold since it corresponds to $90 \%$ of the covered time in the graph because of the nature of the distance formula.

The potential errors in the implementation of our approach threaten the validity of our results. We benefited from a stable graph package NetworkX $\mathrm{X}^{2}$ in our operations and used its methods whenever possible, for example, betweenness centrality calculations and DFS for finding paths between developers. To prevent potential bugs, we performed multiple code review sessions with a third researcher. Also, we shared the implementation online ${ }^{8}$ for replicability of the results.

We used developer comments in issue tracking systems to validate our approach, however, we do not claim that the number of comments shows the key developers in a project. We just claim that there should be a correlation between the top commenters and the key developers in the same time period. Then, we used this idea to show that our approach produced more logical results than the random case with a Monte Carlo simulation.

\footnotetext{
${ }^{10}$ http://siemens.github.io/codeface/icse2017/ (Accessed on 13 Aug 2020)
} 
External validity concerns about generalization of the findings in studies [24]. In our case studies, we used three different OSS projects. Even though we did not conduct a case study in an industrial company, we selected projects from Apache ${ }^{11}$, a 20-year established foundation. Also, the sizes of the projects are different as seen in Table 2. Although we believe that we have enough data for an initial assessment, in the future, we need to run our algorithms in more OSS and industrial datasets.

\section{CONCLUSION AND FUTURE WORK}

In this study, we proposed different categories for key developers in software development projects: jacks, mavens and connectors. To identify the developers in these subcategories of key developers, we proposed separate algorithms. Then, we conducted case studies on three OSS projects (Hadoop, Hive and Pig). Since there was no labeled data for the key developer categories, we used developers' comments in issue tracking systems to validate our results. The key developers found by our model were compatible with the top commenters up to $92 \%$. The results indicated that our approach has promising results to identify key developers in software projects. We can summarize the contributions of our study as follows:

- We offered a novel categorization for the key developers inspired by The Tipping Point from Gladwell [15].

- For each of the three key developer categories (jacks, mavens and connectors), we proposed the corresponding algorithms using a traceability graph (network) of software artifacts.

- The findings of this study might shed light on the truckfactor problem. Key developers can be used to find the truck factor of the projects.

- Identifying key developers in a software project might help the software practitioners for making managerial decisions.

As future work, we plan to run our algorithms on the projects in industrial datasets and validate our results by interviewing project stakeholders and creating a labeled dataset for these types of key developers. We are also planning to use the artifact traceability graph to categorize projects (balanced or hero) and recommend replacements for leaving developers.

\section{REFERENCES}

[1] Amritanshu Agrawal, Akond Rahman, Rahul Krishna, Alexander Sobran, and Tim Menzies. 2018. We don't need another hero?: the impact of heroes on software development. In Proceedings of the 40th International Conference on Software Engineering: Software Engineering in Practice. ACM, 245-253.

[2] Mohammad Y Allaho and Wang-Chien Lee. 2013. Analyzing the social ties and structure of contributors in open source software community. In Proceedings of the 2013 IEEE/ACM International Conference on Advances in Social Networks Analysis and Mining. 56-60.

[3] Guilherme Avelino, Eleni Constantinou, Marco Tulio Valente, and Alexander Serebrenik. 2019. On the abandonment and survival of open source projects: An empirical investigation. In 2019 ACM/IEEE International Symposium on Empirical Software Engineering and Measurement (ESEM). IEEE, 1-12.

[4] Guilherme Avelino, Leonardo Passos, Andre Hora, and Marco Tulio Valente. 2016 A novel approach for estimating truck factors. In 2016 IEEE 24th International Conference on Program Comprehension (ICPC). IEEE, 1-10.

[5] Christian Bird, Alex Gourley, Prem Devanbu, Michael Gertz, and Anand Swaminathan. 2006. Mining email social networks. In Proceedings of the 2006 international workshop on Mining software repositories. 137-143.

[6] Ulrik Brandes. 2001. A faster algorithm for betweenness centrality. Fournal of mathematical sociology 25, 2 (2001), 163-177.
[7] H Alperen Cetin. 2019. Identifying the most valuable developers using artifact traceability graphs. In Proceedings of the 2019 27th ACM foint Meeting on European Software Engineering Conference and Symposium on the Foundations of Software Engineering. 1196-1198.

[8] Jinghui Cheng and Jin LC Guo. 2019. Activity-based analysis of open source software contributors: roles and dynamics. In 2019 IEEE/ACM 12th International Workshop on Cooperative and Human Aspects of Software Engineering (CHASE). IEEE, 11-18.

[9] Valerio Cosentino, Javier Luis Cánovas Izquierdo, and Jordi Cabot. 2015. Assessing the bus factor of Git repositories. In 2015 IEEE 22nd International Conference on Software Analysis, Evolution, and Reengineering (SANER). IEEE, 499-503.

[10] Kevin Crowston, Kangning Wei, Qing Li, and James Howison. 2006. Core and periphery in free/libre and open source software team communications. In Proceedings of the 39th Annual Hawaii International Conference on System Sciences (HICSS'06), Vol. 6. IEEE, 118a-118a.

[11] Enrico Di Bella, Alberto Sillitti, and Giancarlo Succi. 2013. A multivariate classification of open source developers. Information Sciences 221 (2013), 72-83.

[12] Mívian Ferreira, Thaís Mombach, Marco Tulio Valente, and Kecia Ferreira. 2019. Algorithms for estimating truck factors: a comparative study. Software Quality Journal 27, 4 (2019), 1583-1617.

[13] Linton C Freeman. 1978. Centrality in social networks conceptual clarification. Social networks 1, 3 (1978), 215-239.

[14] Thomas Fritz, Gail C Murphy, Emerson Murphy-Hill, Jingwen Ou, and Emily Hill. 2014. Degree-of-knowledge: Modeling a developer's knowledge of code. ACM Transactions on Software Engineering and Methodology (TOSEM) 23, 2 (2014), $1-42$.

[15] Malcolm Gladwell. 2006. The tipping point: How little things can make a big difference. Little, Brown.

[16] Mitchell Joblin, Sven Apel, Claus Hunsen, and Wolfgang Mauerer. 2017. Classifying developers into core and peripheral: An empirical study on count and network metrics. In 2017 IEEE/ACM 39th International Conference on Software Engineering (ICSE). IEEE, 164-174.

[17] Takeshi Kakimoto, Yasutaka Kamei, Masao Ohira, and Kenichi Matsumoto. 2006. Social network analysis on communications for knowledge collaboration in oss communities. In Proceedings of the International Workshop on Supporting Knowledge Collaboration in Software Development (KCSD'06). Citeseer, 35-41.

[18] Makrina Viola Kosti, Robert Feldt, and Lefteris Angelis. 2016. Archetypal personalities of software engineers and their work preferences: a new perspective for empirical studies. Empirical Software Engineering 21, 4 (2016), 1509-1532.

[19] Audris Mockus. 2010. Organizational volatility and its effects on software defects. In Proceedings of the eighteenth ACM SIGSOFT international symposium on Foundations of software engineering. 117-126.

[20] Gustavo Ansaldi Oliva, José Teodoro da Silva, Marco Aurélio Gerosa, Francisco Werther Silva Santana, Cláudia Maria Lima Werner, Cleidson Ronald Botelho de Souza, and Kleverton Carlos Macedo de Oliveira. 2015. Evolving the system's core: a case study on the identification and characterization of key developers in Apache Ant. Computing and Informatics 34, 3 (2015), 678-724.

[21] Michael Rath and Patrick Mäder. 2019. The SEOSS 33 dataset-Requirements, bug reports, code history, and trace links for entire projects. Data in brief 25 (2019), 104005.

[22] Peter C Rigby and Christian Bird. 2013. Convergent contemporary software peer review practices. In Proceedings of the 2013 9th Foint Meeting on Foundations of Software Engineering. 202-212.

[23] Peter C Rigby, Yue Cai Zhu, Samuel M Donadelli, and Audris Mockus. 2016. Quantifying and mitigating turnover-induced knowledge loss: case studies of Chrome and a project at Avaya. In 2016 IEEE/ACM 38th International Conference on Software Engineering (ICSE). IEEE, 1006-1016.

[24] Per Runeson and Martin Höst. 2009. Guidelines for conducting and reporting case study research in software engineering. Empirical software engineering 14, 2 (2009), 131.

[25] Caitlin Sadowski, Emma Söderberg, Luke Church, Michal Sipko, and Alberto Bacchelli. 2018. Modern code review: a case study at google. In Proceedings of the 40th International Conference on Software Engineering: Software Engineering in Practice. 181-190.

[26] Emre Sülün, Eray Tüzün, and Uğur Doğrusöz. 2019. Reviewer Recommendation using Software Artifact Traceability Graphs. In Proceedings of the Fifteenth International Conference on Predictive Models and Data Analytics in Software Engineering. 66-75.

[27] Jing Wu and Khim Yong Goh. 2009. Evaluating longitudinal success of open source software projects: A social network perspective. In 2009 42nd Hawaii International Conference on System Sciences. IEEE, 1-10.

[28] Minghui Zhou and Audris Mockus. 2012. What make long term contributors: Willingness and opportunity in OSS community. In 2012 34th International Conference on Software Engineering (ICSE). IEEE, 518-528.

\footnotetext{
${ }^{11}$ https://www.apache.org/
} 